\title{
Carcinosarcoma of the ovary: A case report
}

\author{
Abdellah Babahabib ${ }^{1,}$,, Mohamed Elmarjany ${ }^{2}$, Hicham Bakkali ${ }^{3}$, Mehdi Elhassani ${ }^{1}$, \\ Jaouad Kouach $^{1}$, Rhali Driss Moussaoui ${ }^{1}$, Mohamed Dehayni ${ }^{1}$ \\ ${ }^{1}$ Department of Gynecology-obstetrics, Military Training Hospital Med V, Rabat, Morocco \\ ${ }^{2}$ Department of Radiotherapy, Military Training Hospital Med V, Rabat, Morocco \\ ${ }^{3}$ Department of Anesthesiology and Critical Care, Military Training Hospital Med V, Rabat, Morocco
}

\section{Email address:}

babahabib2003@hotmail.com (A. Babahabib), hbakkali@ymail.com (H. Bakkali),kouach_jaouad@yahoo.fr(J. Kouach), Moussaouidriss@Yahoo.fr (R. D. Moussaoui), Mohamedehayni@gmail.com (M. Dehayni)

\section{To cite this article:}

Abdellah Babahabib, Mohamed Elmarjany, Hicham Bakkali, Mehdi Elhassani, Jaouad Kouach, Rhali Driss Moussaoui, Mohamed Dehayni. Carcinosarcoma of the Ovary: A Case Report. Clinical Medicine Research. Vol. 3, No. 4, 2014, pp. 90-93.

doi: $10.11648 /$ j.cmr.20140304.12

\begin{abstract}
The carcinosarcoma is a mixed tumour composed of epithelial and mesenchymatous malignant tissus. It can occur on the level of all the female genital tract, most frequently the uterus. The ovarian localization is rare. The purpose of our work is to discuss the problems of differential diagnosis raised by this rare entity. We report the case of a 60 years old patient, who consulted for an abdominal mass of $20 \mathrm{~cm}$. Tomodensitometry of the abdomen and the pelvic had objectified the right ovarian origin. The patient was treated by total colpohysterectomy with bilateral annexectomy. Through this case with review of the literature we shall discuss the histogenetic assumptions of this entity which are the object of controversies as well as the prognosis and therapeutic implications.
\end{abstract}

Keywords: Carcinosarcoma, Malignant Mixed Mesodermal Tumor, Ovarian Cancer, Chemotherapy

\section{Introduction}

Ovarian carcinosarcomas, also known as malignant mixed mesodermal tumors or malignant mixed müllerian tumors (MMMT), are a rare group of neoplasms composed of a mixture of both sarcomatous and carcinomatous components. It is a rare histologic subtype of ovarian cancer that is diagnosed in $1 \%-4 \%$ of all ovarian cancer survivors [1]. It can originate anywhere along the female genital tract, and in the peritoneum; the most frequent location is the uterus, but they can be found in the ovary, vagina, cervix and fallopian tubes [2]. They affect mainly post menopausal women, with an incidence in younger women of only $10 \%$ [3]. MMMTOs are aggressive tumors and respond poorly to treatment. It is a poorly understood disease. There is no consensus on the optimal therapy because of its rarity and the lack of centralized data collection.

We report a case of ovary carcinosarcoma for it's rarity and prognostic aspects of this tumors.

\section{Case Report}

Our case is about a 60 years old women who had experienced 4 months of painfull abdominal mass with vaginal bleeding. Gynecological exam showed a pelvic mass mesuring $20 \mathrm{~cm}$ in the average diameter. Tomodensitometry of the abdomen and the pelvic showed an ovarian mid-cystic mid-solid mass with an ascitis. The other radiological exam was normal. The tumor marker CA125 was elevated (156 UI/ml). An hysterectomy, an ablation of the ovarian mass and an ommentectomy were carried out. Macroscopic exam showed a fragmented ovarian tumor weigheted $700 \mathrm{~g}$ and mesuring $17 \mathrm{~cm}$ of the large diameter with cystic and solid component with fibrosis, hemorragic and necrosis areas. Histological exam showed a biphasic tumor:

- A sarcomatous one composed by fusiform cells, with myxoid areas and some rare chondroid foci.

- A carcinomatous one composed by irregular gland and a rare foci of squamous differenciation (Figure 1a-b). 


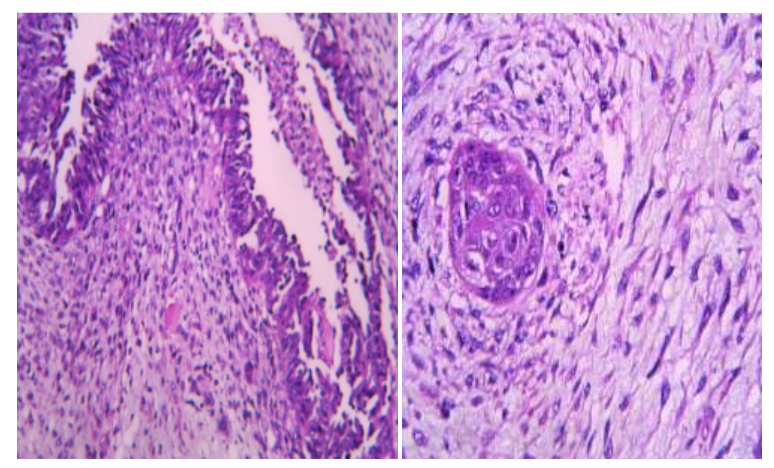

(a)

(b)

Figure 1. tumor composed of both carcinomatous and mesenchymal components (HEx40; HEx100)

Ommentectomy dissection showed numerous metastatic foci from the sarcomatous component. After surgery, an adjuvant chemotherapy were carried out with adriamycine $\left(60 \mathrm{mg} / \mathrm{m}^{2}\right)$ and cisplatine $\left(75 \mathrm{mg} / \mathrm{m}^{2}\right)$ during 6 cycles. The follow up is about 5 months without disease. The tumor marker CA 125 was normal one month after the surgery.

\section{Discussion}

Ovarian carcinosarcomas are rare tumours and pathologically consist of a mixture of epithelial and malignant mesenchymal components. They affect post menopausal women, and the mean age of presentation is 65 years [4]. Our patient was 60 years. Pelvic irradiation may increase the chance of developing an ovarian carcinosarcoma [5].

The clinical presentation of MMMTOs is similar to the epithelial tumors. The main symptoms and signs are abdominal distension, pain, a palpable mass, ascitis, nausea, vomiting and weight loss. Elevated levels of the tumor marker CA125 can be seen in up to $74 \%$ of patients. AFP has been occasionnaly described as a tumor marker.

Ultrasonography usually shows up large tumors. They can be solid or cystic with thick septa. Tomodensitometry and MRI are perfomed to describe the characteristics of the tumor.

The diagnosis of the histologic type is never made preoperatively. Macroscopically the tumours are solid and/or cystic, fleshy, haemorrhagic and frequently spread beyond the ovary [6] as we have seen in our case. Histologically, MMMTOs include two component of epithelial and stromal origin. The sarcomatous component can be classified as homologous (sarcoma of the endometrial stroma, fibrosarcoma, leimyosarcoma), or heterologous, composed of extra ovarian elements (rhabdomyosarcoma, osteosarcoma, liposarcoma, chondrosarcoma) [1]. In our case, we have seen a rare chondroid area. The epithelial component is classified according to subtype and grade. The most common forms are the endometrioid, indifferentiated, and serous papillary $[2,4]$. In our case it's an endometrioid type with squamous differenciation. Undifferentiation is a common feature of both components of the tumor. The high grade indifferentiation of the epithelial component has been described as a poor prognosis factor [1]. Some authors [1] suggested that the tumors with heterologous sarcomal components had a worse prognosis, like our case but the follow up is not enough to evaluated this factor. The sarcomal component of the tumors varies between 15 and 85 percent,but it is predominant in metastase, as we have seen in peritoneal nodule [4]. Immunohistochemical examination: Anti-cytokeratin monoclonal antibodies and anti-epithelial membrane antigene were used for the detection of the epithelial component. To detect the mesenchymal components, avimentin monoclonal antibody, CD10, smooth musle actin, desmine, and myoglobine are the most usefull antobodies. S100 protein polyclonal antibody was used to detect chondroid or adipose tissue differenciation [3]. The stage classification used for carcinosarcoma of the ovary is the same FIGO system that is applied to the other ovarian carcinomas [6].

Histogenesis: 3 theories try to explain the histogenesis of MMMTOs [3]:

- The combination theory suggests that both cellular lines come from the same stem cell.

- The collision theory proposes that two different cellular lines with different mutations generate the tumor.

- The conversion theory suggests that a cellular line already carrying the mutation suffers a metaplasic transformation, producing a further cellular clone.

Advances in our knowledge of tumor histogenesis have documented the monoclonal origin of the majority of cases. As for all malignant tumors, the FIGO classification based on surgical staging is used. In the initial stage (stade I), the treatment is total adnexo-hysterectomy, omentectomy, lymphadenectomy, and the removal of any suspicious mass, as for other ovarian tumors. In advanced stages, cytoreduction surgery is the only treatment, showing longer patient survival.

Recently, the gynaecologic oncology group reported a good response to platinium as a first line treatment [6]. It was the treatment administred to our patient. The role of therapeutic radiotherapy is not established [6]. Maximal effort during the initial surgery is currently the only valid way to treat those cancers. In MMMTOs, the principal prognostic factors are histologic grade, stage, and the residual disease after surgery. The over expression of p53 has been shown to be a weakly correlated with a longer survival, this can be found in both components of carcinosarcoma.The median survival of patients with MMMT of the ovary have been estimated at 8-16 months [2].

No uniform agreement exists about the optimal treatment of these malignancies except that achieving optimal debulking at the time of initial surgery significantly improves overall survival [11]. Most published data are limited to retrospective reviews, and because of the rarity of the disease, few institutions are able to accrue a sufficient number of 
patients for prospective studies. Brown et al. compared prospectively the clinicopathologic features and outcomes of 65 patients with carcinosarcoma of the ovary to 746 patients with serous adenocarcinoma of the ovary. They reported a median survival of 14.8 months in patients with optimally debulked FIGO stage III ovarian carcinosarcomas as opposed to 3.1 months for suboptimally or nondebulked stage III disease. Their data show a significantly lower objective response rate to platinum therapy [12]. However, most authors report a higher response rate for platinum-based regimens than for nonplatinum combinations. Thigpen et al. in a Gynecologic Oncology Group study presented data involving 136 patients with ovarian carcinosarcomas treated with cisplatin $50 \mathrm{mg} / \mathrm{m}^{2}$ every 3 weeks until disease progression or unacceptable toxicity occurred. They concluded that cisplatin is active as initial therapy for these tumors. The median survival in 130 patients was 11.7 months [13]. Baker et al. published a prospective trial that involved 11 advanced or recurrent ovarian carcinosarcoma patients treated with cisplatin, adriamycin, and dacarbazine after surgical debulking. Their estimated 1, 2, and 3 year survival was $70 \%, 35 \%$, and $35 \%$, respectively [14]. Zorzou et al. treated nine patients with aggressive cytoreduction followed by anthracycline-based chemotherapy. Their median overall survival was 32.9 months with no statistical difference between early and advanced stages [15]. Crotzer et al. published a pilot study of cisplatin, ifosfamide, and mesna in the treatment of malignant mixed mesodermal tumors of the ovary. The median survival for the eight patients was 21 months [16]. Rutledge et al. evaluated retrospectively 11 patients with early and advanced disease who received cisplatin $20 \mathrm{mg} / \mathrm{m}^{2}$ and ifosfamide $1.5 \mathrm{gm} / \mathrm{m}^{2}$ for 4 days every 21 days for six cycles. The progression-free interval and overall survival for all stages were improved when compared to 16 patients treated with carboplatin (AUC6) and paclitaxel $175 \mathrm{mg} / \mathrm{m}^{2}$ every 21 days for six cycles. However, this advantage disappeared when only advanced stages were considered [17]. Patsner et al. treated four patients with metastatic ovarian carcinosarcoma with mesna, doxorubicin, ifosfamide, and dacarbazine chemotherapy. Two of the four patients had optimal cytoreduction. They reported two complete responses of 34and 46-month duration [18]. Le et al. reported on 33 patients with metastatic carcinosarcoma of the ovary. At the end of surgery, 22 patients were left with macroscopic residual disease. Adjuvant chemotherapy consisted of cisplatin and doxorubicin. Survival was $35 \%$ at 5 years. Thirteen patients had a second-look laparotomy, and the authors concluded that a second operation offers little helpful information on the management of these tumors. Mok et al. presented a case series of ten patients and reviewed the use of platinum-based combination chemotherapy for ovarian carcinosarcoma at their institution. All patients underwent optimal cytoreductive surgery. Following surgery, all patients received platinum-based chemotherapy and seven patients were treated with ifosfamide $\left(1200 \mathrm{mg} / \mathrm{m}^{2}\right)$ and cisplatin $(75$ $\left.\mathrm{mg} / \mathrm{m}^{2}\right)$. The median survival was 46 months for the entire cohort (two patients with stage IIC, seven patients with stage IIIC, and one patient with stage IV). They concluded that platinum-based combination chemotherapy after optimal cytoreductive surgery may be effective in the treatment of these tumors. They also noted that the toxicities of these regimens, especially those including ifosfamide, are significant [19]. Invariably, a diagnosis of advanced stage carcinosarcoma is associated with a dismal prognosis, and most studies record few survivors at 2 years or even 1 year. The case series presented here reports a more encouraging survival time, especially in the group of patients treated with optimal cytoreduction and adjuvant cisplatin and ifosfamide every 28 days.Administration of cisplatin and ifosfamide requires hospitalization for the duration of treatment, while the carboplatin and paclitaxel combination is infused in an outpatient setting. The latter chemotherapy regimen was administered more commonly in recent years because of the recognition of a monoclonal epithelial origin for uterine carcinosarcomas. Survivals in the two groups presented are comparable to survivals observed in ovarian epithelial malignancies. The groups of patients presented are small but homogeneous, since all cases had metastatic disease found at the initial diagnosis. Survival curves are the only valid way to compare survival. The median survival may be misleading because the patients in the noncisplatin/ifosfamide group actually survive longer at the end. However, this may be just by chance because the number of patients is small. Only two patients in the noncisplatin and ifosfamide group made this group look better overall. The curves do differ initially, and this observation merits further investigation about the possible benefits of cisplatin and ifosfamide combination regimen. However, this data set is showing that the curves actually cross in the end, which makes the statistical comparisons of survival curves less valid. The results of this study were utilized for a power analysis. While the difference in survival between the cisplatin and ifosfamide group and the carboplatin and taxol group would become statistically meaningful (power of $80 \%$ ) at 279 patients in each group, the difference in progression-free interval would achieve significance with only 29 patients in each group. For advanced stage ovarian carcinosarcoma, we recommend optimal cytoreduction and adjuvant chemotherapy. Because of the rarity of the disease, only a nationwide multi-institutional trial can provide a better answer to what constitutes the most effective cytotoxic regimen.

\section{Conclusion}

MMMTOs constitute a rare group tumour, which are highly aggressive and respond poorly to treatment. The diagnosis is based on histoligical identification of both epithelial and sarcomatous component. IHC exam is very helpful to undifferentiated cases. Principal prognosis factors are heterologous sarcomal component and undifferentiated epithelial component. Complete surgery is the best treatment for these tumors. 


\section{References}

[1] Silasi DA, Illuzzi JL, Kelly MG , Rutherford TJ, Mor G, Azodi M, Schwartz PE.. Carcinosarcoma of the ovary. Int J Gynecol Cancer 2008; 18: 22-29.

[2] Navirini R, Pineda RL. Malignant mixed mullerian tumors of the ovary. curr opin obstet gyneco 2006; 18:20-23.

[3] Rauh-Hain JA, Growdon WB, Rodriguez N, Goodman AK, Boruta DM 2nd, Schorge JO, Horowitz NS, del Carmen MG.. Carcinosarcoma of the ovary: a case-control study. Gynecologic.Oncology,2011; 121: 477-481.

[4] Harris MA, Delap LM, Sengupta PS, Wilkinson PM, Welch RS, Swindell R, Shanks JH, Wilson G, Slade RJ, Reynolds K, Jayson GC. Carcinosarcoma of the ovary. Br J Cancer 2003; 88: 654-57.

[5] Mok JE, Kim YM, Jung MH, Kim KR, Kim DY, Kim JH, Kim YT, Nam JH. Malignant mixed müllerian tumors of the ovary: experience with cytoreductive surgery and platinum-based combination chemotherapy. Int $\mathrm{J}$ Gynecol Cancer 2006; 16: 101-105.

[6] Jin Z, Ogata S, Tamura G, Katayama Y, Fukase M, Yajima M, Motoyama T. Carcinosarcomas (malignant mullerian mixed tumors) of the uterus and ovary: a genetic study with special reference to histogenesis. Int J Gynecol Pathol 2003; $22: 368-73$

[7] Wei LH, Huang CY, Cheng SP, Chen CA, Hsieh CY. Carcinosarcoma of ovary associated with previous radiotherapy. Int J Gynecol Cancer 2001; 11: 81-84.

[8] Tate TJ, Blessing JA, DeGeest K, Look KY, Homesley HD. Cisplatin as initial chemotherapy in ovarian carcinosarcomas : a Gynecologic Oncology Group Study. Gynecol Oncol 2004; 93: 336-39.

[9] Cicin I, Saip P, Eralp Y, Selam M, Topuz S, Ozluk Y, Aydin Y, Topuz E. Ovarian carcinosarcomas: clinocopathological prognostic factors and evaluation of chemotherapy regimens containing platinum. Gynecol Oncol 2008; 108: 136-40.

[10] Rutledge TL, Gold MA, McMeekin DS, Huh WK, Powell MA, Lewin SN, Mutch DG, Johnson GA, Walker JL, Mannel
RS. Carcinosarcoma of the ovary - a case series. Gynecol Oncol 2006; 100: 128-32.

[11] Paulsson G, Andersson S, Sorbe B. A population-based series of ovarian carcinosarcomas with long-term follow-up. Anticancer Research, 2013; 3: 1003-1008.

[12] Brown E, Stewart M, Rye T, Al-Nafussi A, Williams AR, Bradburn M, Smyth J, Gabra H. Carcinosarcoma of the ovary: 19 years of prospective data from a single center. Cancer 2004;100: 2148-53.

[13] Thigpen J, Blessing JA, DeGeest K, Look KY, Homesley HD; GynecologicOncology Group. Cisplatin as initial chemotherapy in ovarian carcinosarcomas: a Gynecologic Oncology Group study. Gynecol Oncol 2004;93:336-9.

[14] Chun KC, Kim JJ, Kim DY et al. Optimal debulking surgery followed by paclitaxel/platinum chemotherapy is very effective in treating ovarian carcinosarcomas: a single center experience. Gynecologic and Obstetric Investigation 2011; 72: 208-214,

[15] Zorzou MP, Markaki S, Rodolakis S et al. Clinicopathological features of ovarian carcinosarcomas: a single institution experience. Gynecol Oncol $2005 ; 96: 136-42$

[16] Crotzer DR, Wolf JK, Jenkins AD, Gershenson DM, Levenback C. Apilot study of cisplatin, ifosfamide and mesna in the treatment of malignant mixed mesodermal tumors of the ovary. Proc Am Soc Clin Oncol 2003;22:474.

[17] Rutledge TL, Gold MA, McMeekin DS et al. Carcinosarcoma of the ovary - a case series. Gynecol Oncol 2006;100:128-32.

[18] Signorelli M, Chiappa V, Minig L, Fruscio R, Perego P, Caspani G, Battistello M, Colombo N.Platinum, anthracycline,and alkylating agent-based chemotherapy for ovarian carcinosarcoma. International Journal of Gynecological Cancer 2009; 19: 1142-1146.

[19] Mok JE, Kim YM, Jung MH, Kim KR, Kim DY, Kim JH, Kim YT, Nam JH.. Malignant mixed mullerian tumors of the ovary: experience with cytoreductive surgery and platinumbased combination chemotherapy. Int J Gynecol Cancer 2006; $16: 101-5$. 